\title{
Multidetector computed tomography of chest trauma: indications, technique and interpretation
}

\author{
Hynek Mirka $\cdot$ Jiri Ferda $\cdot$ Jan Baxa
}

Received: 22 January 2012 / Accepted: 10 July 2012 /Published online: 4 August 2012

(C) The Author(s) 2012. This article is published with open access at Springerlink.com

\begin{abstract}
Background Chest traumas are a significant cause of mortality and morbidity, especially in the younger population. Methods Diagnostic imaging plays a key role in their management. Multidetector computed tomography (MDCT) is the most important imaging method in this field. Its advantages include especially high speed and high geometric resolution in any plane.

Results The method allows us to view large parts of the body with minimal motion artifacts and to create accurate multiplanar and three-dimensional (3D) reformations, which make the diagnosis significantly more accurate. Because of its advantages MDCT has become the first-choice method in high-energy traumas.

Conclusion This article summarises the position of MDCT in the diagnostic algorithm of chest injuries, technical aspects of the examination and imaging findings in traumas of the individual chest compartments.

Teaching Points

- Diagnostic imaging plays a key role in the management of high-energy chest trauma.

- MDCT is the most important imaging method in this kind of injury, as detailed information can be acquired in a short acquisition time.

- Multiplanar and three-dimensional (3D) reformattings make the diagnosis significantly more accurate.
\end{abstract}

Keywords Multidetector computed tomography · Thorax · Wounds and injuries $\cdot$ Blunt trauma $\cdot$ Penetrating trauma

H. Mirka $(\bowtie) \cdot$ J. Ferda $\cdot$ J. Baxa

Department of Imaging methods,

Charles University and University Hospital in Pilsen,

Alej Svobody 80,

30460 Pilsen, Czech Republic

e-mail: mirka@fnplzen.cz

\section{Introduction}

In industrialised countries injuries represent a significant socioeconomic problem. They commonly occur in the younger population and are the most common cause of death in people between 25 and 44 years of age. Chest injuries occur in about $20 \%$ of all trauma patients. In up to $80 \%$ of cases they are associated with injuries of other body parts such as the head (69\%), abdomen and pelvis (43\%) and extremities $(52 \%)[1,2]$. Diagnostic imaging plays a key role in deciding on the therapeutic procedure and determining the prognosis [3]. Multidetector computed tomography (MDCT) is considered to be the most effective imaging method in this field and therefore should be an integral part of the emergency department [4]. This article summarises the position of MDCT in the diagnostic algorithm, examination technique and findings in injuries of the individual chest compartments.

\section{Mechanisms of injury, classification}

Chest injuries usually occur in car crashes, falls from heights, sports injuries and violent acts. The most affected compartments include the chest wall (>50\%), pleura (50\%) and lungs (30-70\%). Rare but often very severe are traumas of the airways ( 2.8 to $5.4 \%$ ), diaphragm (0.4-1.5\%), large vessels $(1.1$ to $2.2 \%)$ and heart $(10 \%)[3,5]$. According to the underlying cause, the injuries are classified as blunt $(90 \%)$ and penetrating (10\%) injuries. Blunt injury is caused by three basic mechanisms, which are often combined. These are a direct impact, compression and deceleration [4]. Direct impact on the chest causes a localised injury of the chest wall at the point of contact. When exposed to major forces, the energy can affect the deeper structures located in their trajectory, such as lungs, heart, blood 
vessels, mediastinum, liver or spleen. Compression causes pressing of the organs against the spine or chest wall and their contusion or rupture. The pulmonary parenchyma, pleura, diaphragm and tracheobronchial tree are often affected. Deceleration causes movement of the organs and shearing forces at the site where they are fixated. This generates traumas of the tracheobronchial tree, aorta, heart and diaphragm (Fig. 1). The causes of a penetrating trauma are often stab and gunshot wounds in victims of violence and war. Due to the higher incidence of vascular and heart injuries, a penetrating trauma has a higher mortality compared to a blunt trauma [6].

\section{Imaging methods}

Clinical symptoms of chest injuries are diverse and often do not correlate with their severity. This is the reason why diagnostic imaging belongs among the first procedures performed after admission to a medical facility. The simplest and fastest feasible methods include chest radiography and ultrasound. They can, especially in unstable patients, provide important information about the presence of serious injuries requiring an emergency intervention, such as tension pneumothorax, large hemothorax, hemopericardium, hemoperitoneum and injuries of the abdominal organs under the diaphragm. These methods are less sensitive for some types of trauma, and therefore, especially in high-energy injuries they cannot be considered as conclusive. Chest radiography is performed in severe injuries in supine position, which further reduces its contribution. Detection of a contusion and laceration of the pulmonary parenchyma, but also a smaller hemothorax and pneumothorax, is also an issue. It is also very unreliable in detecting injuries of the heart and great vessels [7-11]. In addition to low sensitivity in certain types of traumas, its low specificity is a problem. A symptom of expanded mediastinum, which may be a sign of trauma of the major vessels and heart, can be an example.

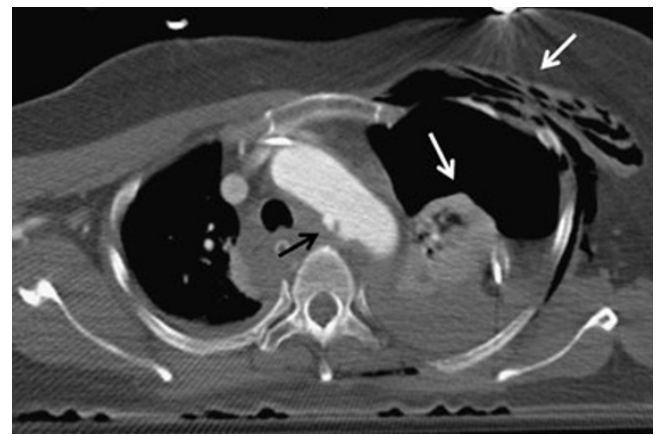

Fig. 1 Combination of two traumatic mechanisms, pedestrian knocked down by a car. Rib fractures, hemothorax and pneumothorax on the left side are results of direct impact (white arrows). Small aortic pseudoaneurysm is an effect of deceleration (black arrow)
However, this concerns only about $20 \%$ of cases. The remaining findings are caused by bleeding without trauma of the major blood vessels or also by non-traumatic causes such as mediastinal lipomatosis, congenital abnormalities, enlarged lymphatic nodes or tumors $[5,12]$. Compared to this, MDCT enables sufficiently accurate assessment of all compartments of the chest and also reveals changes that are not detectable by other methods (Fig. 2). Additional findings are very common in MDCT (up to $83 \%$ of cases), but in fact only some of them can change the therapeutic process (7-41\%) [10, 13-15].

\section{MDCT}

Implementation of MDCT has significantly increased the accuracy of diagnosis of injuries, not only in the area of the chest, and is now considered as a fundamental method in trauma radiology. It is able to comprehensively examine all structures of the chest with a sensitivity and specificity approaching $100 \%$. Excellent spatial and temporal resolution is the main benefit. Because of the isotropic data
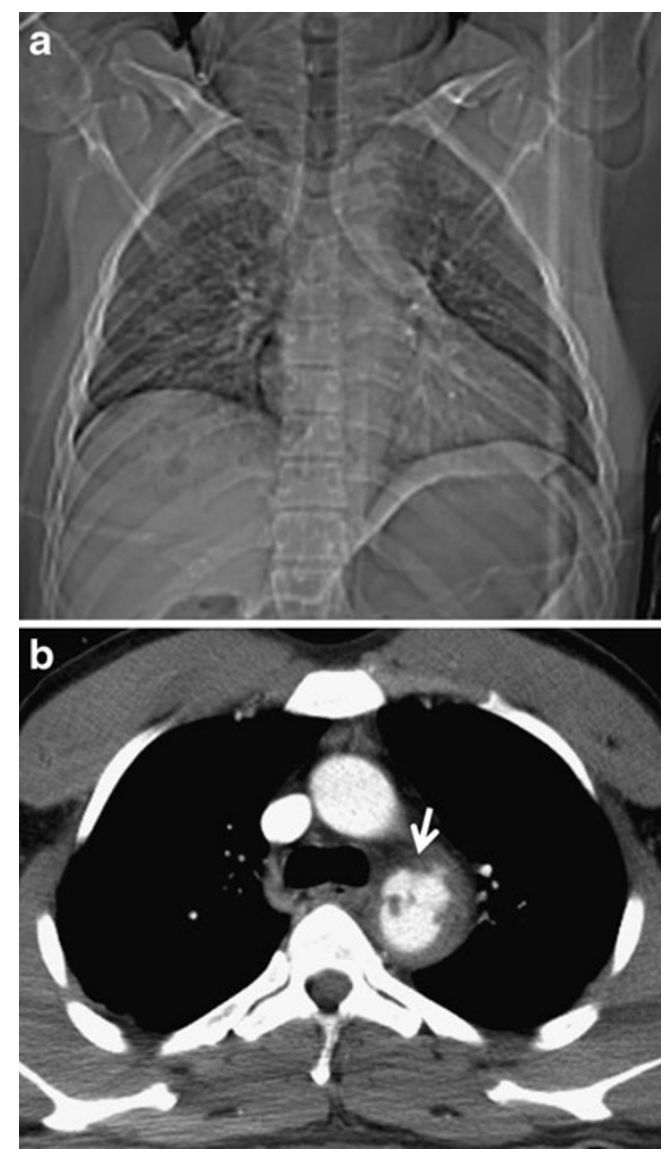

Fig. 2 a, b Patient with laceration of the aorta. a Topogram (analogical to chest radiograph) shows normal mediastinal width. b MDCT reveals rupture of the aortic isthmus with small periaortic hematoma (arrow) 
field, it allows performing two-dimensional (2D) and threedimensional (3D) reformations in any plane and angle of view without loss of geometric resolution and to evaluate the anatomical structures, which are located adversely to the axial plane. A high-speed examination is needed to view the entire examination area in the proper postcontrast circulation phase and to minimise motion artifacts (Fig. 3) [4, 16, 17].

\section{Diagnostic algorithm}

The choice of a diagnostic procedure depends on the patient's condition and traumatic mechanism. In lowenergy traumas (falls from heights of up to $3 \mathrm{~m}$ and traffic accidents at speeds of up to $50 \mathrm{~km} / \mathrm{h}$ ), the standard method includes X-ray and ultrasound examinations. The chest MDCT examination should be performed only in case of unclear findings or if more detailed assessment is needed. In patients with high-energy trauma (falls from heights above $3 \mathrm{~m}$ and traffic accidents at speeds exceeding $50 \mathrm{~km} / \mathrm{h}$ ) and unknown mechanism of trauma, the chest MDCT examination should be performed as a screening method. The examination is usually a part of the whole-body CT. In stable patients with no apparent need of emergency intervention, the MDCT examination can be performed directly after admission to a health-care facility and primary clinical examination. In the case of circulatory instability or if pharmacological circulatory support is needed, the chest $\mathrm{X}$-ray in supine position is usually performed as well as ultrasound examination to exclude findings that require an immediate intervention. The MDCT examination should be performed only after these necessary procedures have been completed [18]. In the event that MDCT is a part of the emergency department and the examination can be performed concurrently with resuscitation, this step can be skipped, and the MDCT can be used in this situation as the primary imaging method (Fig. 4). Routine use of MDCT in cases of high-energy trauma is associated with higher costs, radiation burden and numerous minor additional findings. Nevertheless, this procedure cannot be abandoned because there is a risk of omission of a clinically silent, curable life-threatening condition, such as aortic trauma [10].

\section{Examination technique}

Examination protocols vary for different devices according to their technical parameters. Generally speaking, in order to minimise motion artifacts high examination speed should be used in traumas, and because of the need to perform reformations in other planes the highest resolution in the Z-axis should be used as well. The chest is an area with lower absorption of radiation and higher contrast between the single structures; therefore, in examination focused on the thorax a lower value of the exposure parameters can be used compared with the whole-body examination in which the abdominal area must be taken into account. It is suitable to apply dose modulation systems adjusting the voltage and current values according to a patient's habitus and extent of absorption in the examined area [19]. Care concerning the radiation dose is especially important in children where we can reduce the burden from five to ten times compared with adults by using the appropriate exposure parameters. Dose reduction is achieved not only by using automatic dose modulation, but also by reduction of reference exposure values according to body weight. The kilovolt value can be reduced to $80-100$, and the $\mathrm{mAs}$ value can be decreased to $30-80$ (Fig. 5) [20]. If cardiac and thoracic aorta
Fig. 3 a, b Effect of acquisition speed on the quality of 3D images. a Six-row MDCT, acquisition time $20 \mathrm{~s}$ : significant motion artifacts on the heart and aorta. b Sixty-four-row MDCT, acquisition time $7 \mathrm{~s}$ : heart and aorta are free of artifacts
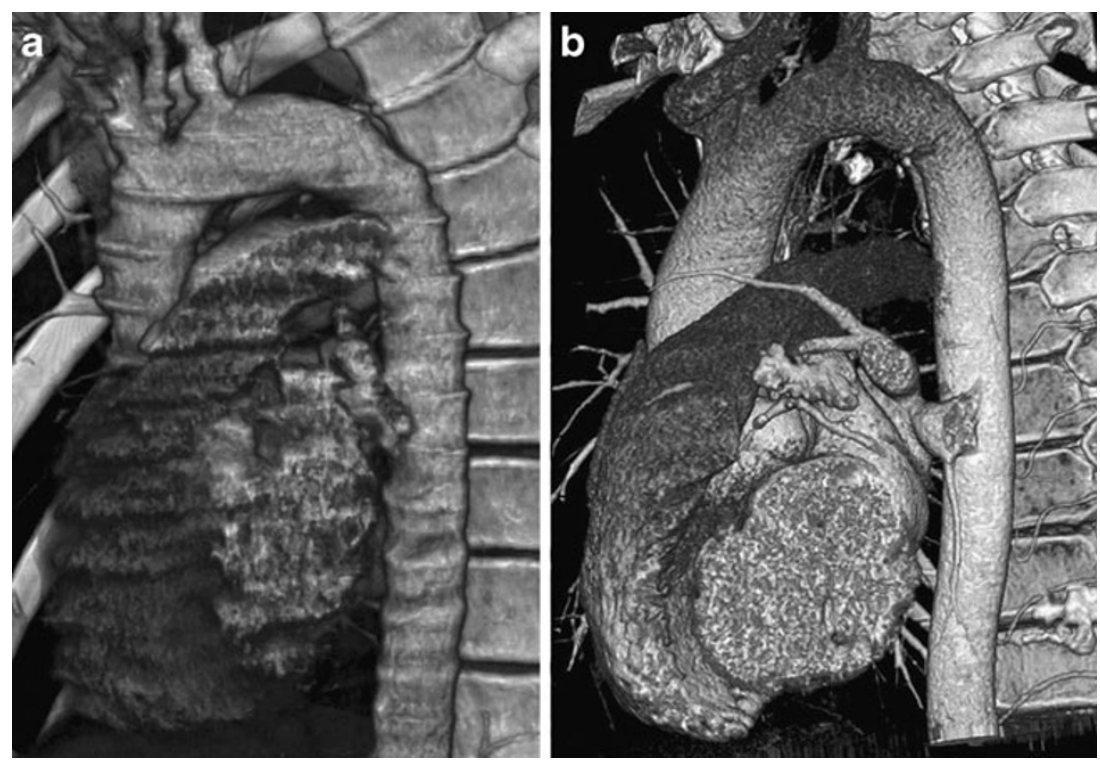


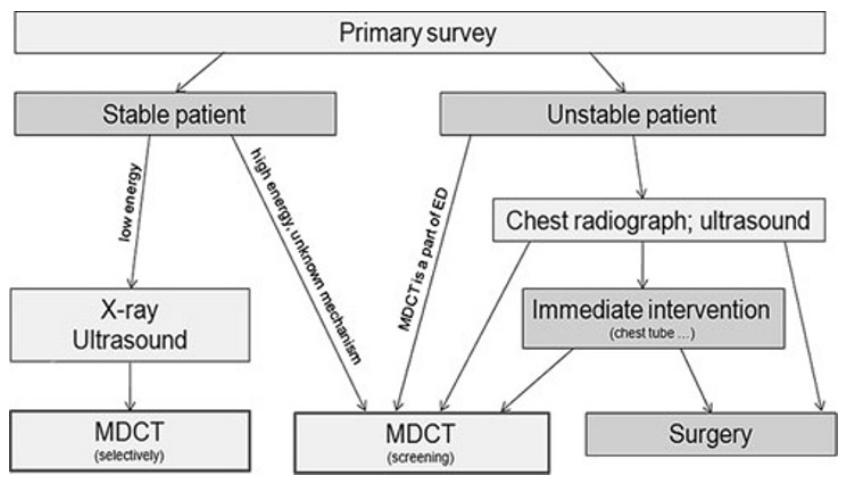

Fig. 4 Diagnostic algorithm used in our institution (ED, emergency departement)

(especially a. ascendens) trauma is suspected, we can use ECG synchronisation to eliminate motion artifacts [21]. If it is possible with regard to the patient's condition, the examination should always be performed with elevated upper limbs.

Application of contrast media is essential for the assessment of vascular structures and parenchymal organs and for detection of active bleeding. Therefore, it is recommended to set a longer scanning delay (30-40 s.) compared with standard chest examinations (Fig. 6). [22, 23]. Examination
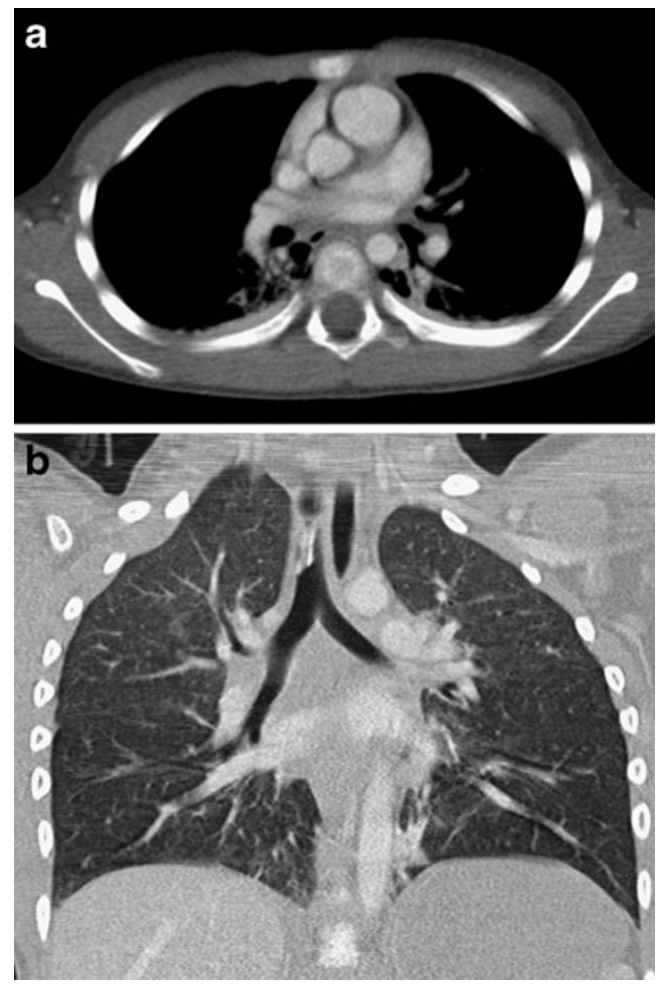

Fig. 5 a, b Low-dose examination in a 6-year-old child. Significant dose reduction was achieved while maintaining an acceptable diagnostic quality $(80 \mathrm{KV}, 40$ effective $\mathrm{mAs}$, dose-length product $37 \mathrm{mGy} * \mathrm{~cm})$. a Axial slice, smooth reconstruction algorithm. b Coronal reformation, edge enhancement reconstruction algorithm
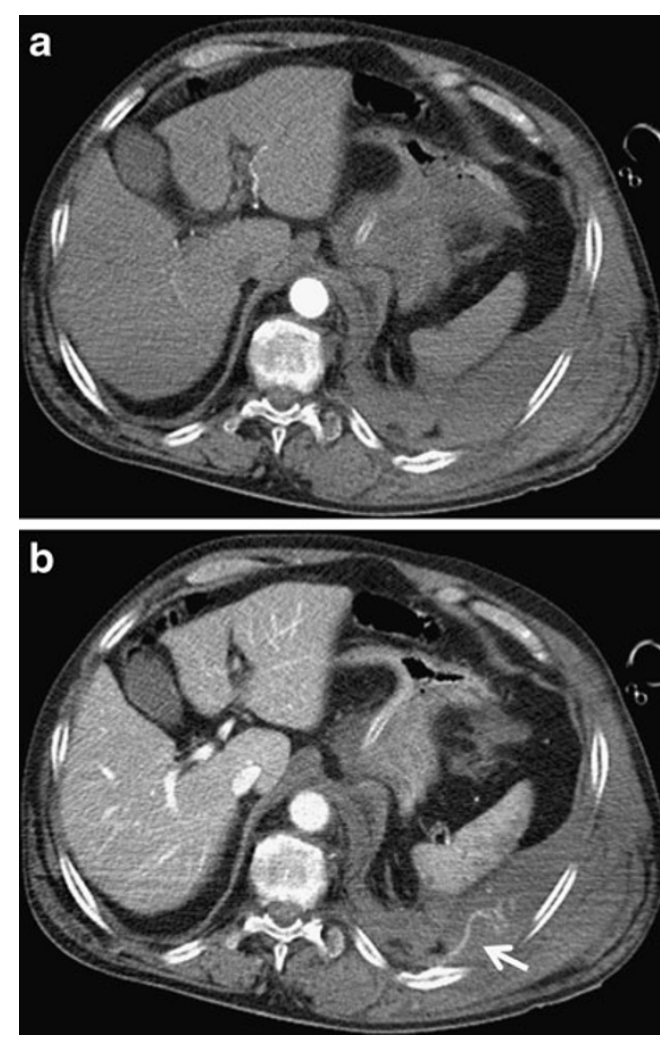

Fig. 6 a, b Active bleeding into the extrapleural hematoma, importance of extended scanning delay after contrast injection. a Scanning delay 20 s: no evidence of contrast material extravasation. b Scanning delay $40 \mathrm{~s}$ : extravasation of contrast material from the intercostal artery (arrow)

of the chest trauma is usually performed without oral contrast agent application. A mall amount of contrast material can be used in case of suspected rupture of the esophagus, usually in addition to standard examination in unclear findings. In this case water-soluble iodinated contrast medium (usually $5-10 \%$ solution) must be used, which does not cause complications and does not affect subsequent surgical therapy. Non-contrast examination in the primary diagnosis is not essential, but it can be used for follow-up examinations focused on the state of the lungs, bronchial tree or skeleton.

The slices reconstructed in three principal planes (axial, coronal and sagittal) or additional reconstructions in other planes and 3D images should be used for evaluation. To view the soft tissues, slices with a width of 3-5 mm reconstructed using a smooth resolution kernel are usually used. In lung parenchyma and bones, we use thinner slices (1$1.5 \mathrm{~mm}$ ) reconstructed using a high-resolution kernel. A series of thin slices with a width of $0.6-1.5 \mathrm{~mm}$ with overlapping of about $1 / 4-1 / 3$ (so-called secondary raw data) should be used for other $2 \mathrm{D}$ and $3 \mathrm{D}$ reformations. Using advanced software and powerful hardware 3D reformations are not time consuming. Their main contribution is a 
synoptic display of traumatic lesions of the skeleton, blood vessels or bronchial tree. In fact, they are not required for diagnosis alone, but may simplify assessment of anatomical relationships and communication with clinicians.

Imaging protocols for thoracic trauma are summarised in Tables 1 and 2. Table 1 shows protocols for different devices that are applicable in primary MDCT examinations. Table 2 summarises modifications of standard protocol depending on clinical situation and purpose of the examination.

\section{Chest wall injuries}

Chest wall injuries are very common. Fractures reflect the intensity and direction of forces during trauma. However, the extent of a chest wall injury does not have to correlate with the injuries of the intrathoracic organs. This is evident especially in children and young adults with a flexible skeleton in whom severe visceral traumas may occur despite there being no fractures.

Table 1 Chest trauma protocols for contemporary MDCT devices

\begin{tabular}{|c|c|c|c|}
\hline & $10-16$ rows & $40-64$ rows & $>64$ rows \\
\hline \multicolumn{4}{|l|}{ Scanning parameters } \\
\hline Voltage $(\mathrm{kV})^{\mathrm{a}}$ & 120 & 120 & 120 \\
\hline Current (eff. mAs) ${ }^{\mathrm{a}}$ & $80-120$ & $80-120$ & $80-120$ \\
\hline Rotation time (s) & 0.5 & $0.33-0.5$ & $0.27-0.5$ \\
\hline Collimation (mm) & $0.75-1.5$ & $0.6-1.2$ & 0.6 \\
\hline Pitch & $1-1.5$ & $0.9-1.5$ & $0.9-1.5$ \\
\hline Scan duration time (s) & $5-10$ & $2-5$ & $1-3$ \\
\hline Scan direction & Craniocaudal & Craniocaudal & Craniocaudal \\
\hline Scan coverage & Lung apex to L1 & Lung apex to L1 & Lung apex to L1 \\
\hline \multicolumn{4}{|c|}{ Intravenous contrast injection } \\
\hline Volume (ml) & 80 & 80 & 80 \\
\hline Concentration (mgI/ml) & $350-400$ & $350-400$ & $350-400$ \\
\hline Flow rate $(\mathrm{ml} / \mathrm{s})$ & $2.5-3$ & $3-4$ & $3-4$ \\
\hline Iodine flux (mgI/ml) & $1,000-1,200$ & $1,200-1,400$ & $1,200-1,600$ \\
\hline Saline flush $(\mathrm{ml} ; \mathrm{ml} / \mathrm{s})$ & $50 ; 2.5-3$ & $50 ; 3-4$ & $50 ; 3-4$ \\
\hline Scan delay $(\mathrm{s})^{\mathrm{b}}$ & $\begin{array}{l}\text { Bolus triggering in } \\
\text { ascending aorta } \\
\text { (threshold } 80 \mathrm{HU} \text { ) } \\
+15 \text { or } 30-40\end{array}$ & $\begin{array}{l}\text { Bolus triggering in } \\
\text { ascending aorta } \\
\text { (threshold } 100 \mathrm{HU} \text { ) } \\
+15 \text { or } 30-40\end{array}$ & $\begin{array}{l}\text { Bolus triggering in } \\
\text { ascending aorta } \\
\text { (threshold } 100 \mathrm{HU} \text { ) } \\
+15 \text { or } 30-40\end{array}$ \\
\hline \multicolumn{4}{|l|}{ Reconstructed images } \\
\hline \multicolumn{4}{|l|}{ Soft tissue } \\
\hline - Slice thickness (mm) & $3-5$ & $3-5$ & $3-5$ \\
\hline - Increment & $3-5$ & $3-5$ & $3-5$ \\
\hline - Recon. algorithm & Smooth & Smooth & Smooth \\
\hline - Orientation & Axial, coronal, sagittal & Axial, coronal, sagittal & Axial, coronal, sagittal \\
\hline \multicolumn{4}{|l|}{ Lungs, bones } \\
\hline - Slice thickness (mm) & $1-1.5$ & $1-1.5$ & $1-1.5$ \\
\hline - Increment & $1-1.5$ & $1-1.5$ & $1-1.5$ \\
\hline - Recon. algorithm & High resolution & High resolution & High resolution \\
\hline - Orientation & Axial, coronal, sagittal & Axial, coronal, sagittal & Axial, coronal, sagittal \\
\hline \multicolumn{4}{|l|}{ Secondary raw data } \\
\hline - Slice thickness (mm) & $0.75-1.5$ & $0.6-1.5$ & $0.6-1.5$ \\
\hline - Increment (mm) & $0.5-1$ & $0.4-1$ & $0.4-1$ \\
\hline - Recon. algorithm & Smooth, high resolution & Smooth, high resolution & Smooth, high resolution \\
\hline - Orientation & Axial & Axial & Axial \\
\hline
\end{tabular}

Protocols are based on the experience of the authors with Siemens equipment; they are universally applicable in each thoracic trauma

${ }^{a}$ Using automatic dose modulation recommended

${ }^{\mathrm{b}}$ Use of bolus triggering preferred 
Table 2 Modification of a standard chest trauma MDCT protocol in different clinical situations

$\begin{array}{ll}\text { Chest MDCT as a part of the whole-body examination } & \text { Higher current value (adequate for examination of the abdomen) } \\ & \text { Contrast media volume } 100 \mathrm{ml} \text {, flow rate } 3-4 \mathrm{ml} / \mathrm{s} \\ & \text { Trigger in descending aorta above the diaphragm (100 HU, delay } 15 \mathrm{~s}) \text { or scanning delay } \\ & 30-40 \mathrm{~s} \\ & \text { Contrast media volume } 80 \mathrm{ml} \text {, flow rate } 4-5 \mathrm{ml} / \mathrm{s} \\ & \text { Trigger in ascending aorta }(80-100 \mathrm{HU} \text {, minimal possible delay-usually } 4-5 \mathrm{~s}) \\ & \text { Optionally ECG triggering (examination of ascending aorta or heart) } \\ \text { Vascular trauma } & \text { Oblique reformated images in the plane of the aortic arch } \\ & \text { Optionally } 3 \mathrm{D} \text { reconstructions (volume rendering, MIP) } \\ & \text { Optionally } 3 \mathrm{D} \text { reconstructions (virtual endoscopy, MinIP) } \\ & \text { Optionally oral contrast agent (5-10\% solution of iodinated contrast material) } \\ & \text { Optionally non-contrast } \\ \text { Bronchial tree trauma } & \text { Optionally low-dose examination (20-50 eff. mAs) } \\ \text { Esophageal trauma } & 80-100 \mathrm{kV} \\ \text { Follow-up examination focused on lungs, bones or } & 20-80 \text { eff. mAs } \\ \text { bronchi } & \text { Contrast media volume } 1-2 \mathrm{ml} / \mathrm{kg} \\ \text { Children } & \text { Flow rate dependent on needle size (22 } \mathrm{G}, 1.5 \mathrm{ml} / \mathrm{s} ; 20 \mathrm{G}, 2-3 \mathrm{ml} / \mathrm{s} ; 18 \mathrm{G}, 3-4 \mathrm{ml} / \mathrm{s})\end{array}$

${ }^{\text {a }}$ According to body weight, in children with body weight more than $54 \mathrm{~kg}$ this is the recommended protocol for adults

\section{Ribs}

Fractures of the ribs are the most common chest wall traumas. They affect approximately $50 \%$ of patients and in fact are usually not clinically significant. However, their impact on adjacent intrathoracic and intraabdominal organs can be serious. In the case of a multiple fracture involving at least three consecutive ribs, a chest wall instability may occur that is associated with an impaired ventilation mechanism and increased risk of atelectasis (flail chest). The first three ribs are well protected by the collarbones, shoulder blades and muscles. Therefore, their fractures suggest a high-energy trauma. They may be associated with injury to the adjacent blood vessels or brachial plexus. In fractures of the lower three ribs, it is necessary to look for liver, spleen and kidney injuries. MDCT can determine the number of fractures, their location and degree of dislocation more accurately than X-rays. Contrary to X-rays, it is also able to detect fractures of the rib cartilages (Fig. 7) [3, 8].

\section{Scapula}

Fracture of the scapula belongs to the commonly overlooked injuries. It is usually caused by strong direct impact or the effect of large axial forces. In up to $40 \%$ of cases, it is associated with pulmonary contusion, pneumothorax or hemothorax [24, 25]. The areas of the scapular body and neck are mostly affected (Fig. 8).

\section{Sternum}

Sternal fractures occur in $8-10 \%$ of cases involving a blunt chest trauma. They are usually caused by direct impact on the anterior chest wall (typically impact with the steering wheel). They are often accompanied by retrosternal hematoma. Heart contusion is also present in $20-40 \%$ of cases. Trauma of the mediastinal vessels can also be present. The retrosternal hematoma is usually separated from the aorta by a strip of fat. This symptom makes differentiation of the hematoma of aortal origin possible. A non-dislocated sternal fracture can be overlooked at the axial scans, and therefore sagittal reformations have the greatest importance for the diagnosis (Fig. 9) [3, 16, 26].

\section{Clavicle}

Clavicle fractures are usually obvious from the clinical examination. MDCT is useful, especially in the diagnosis

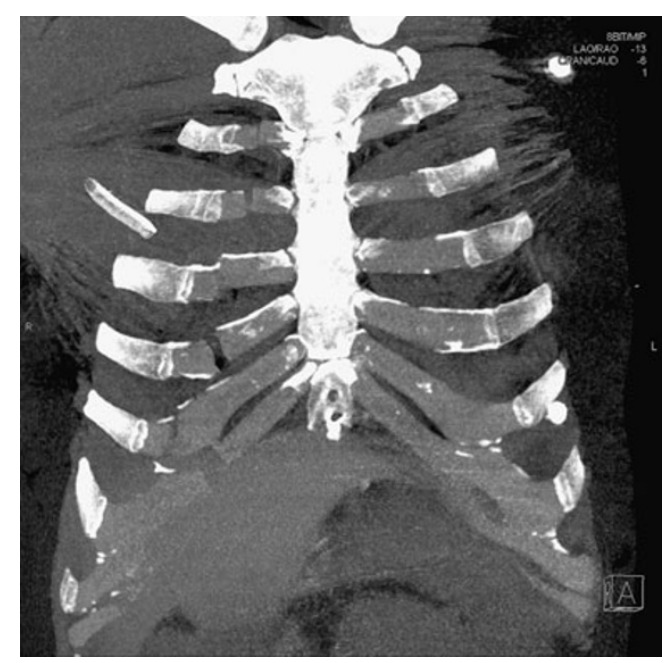

Fig. 7 Multiple rib cartilage fractures. Coronal maximum intensity projection 


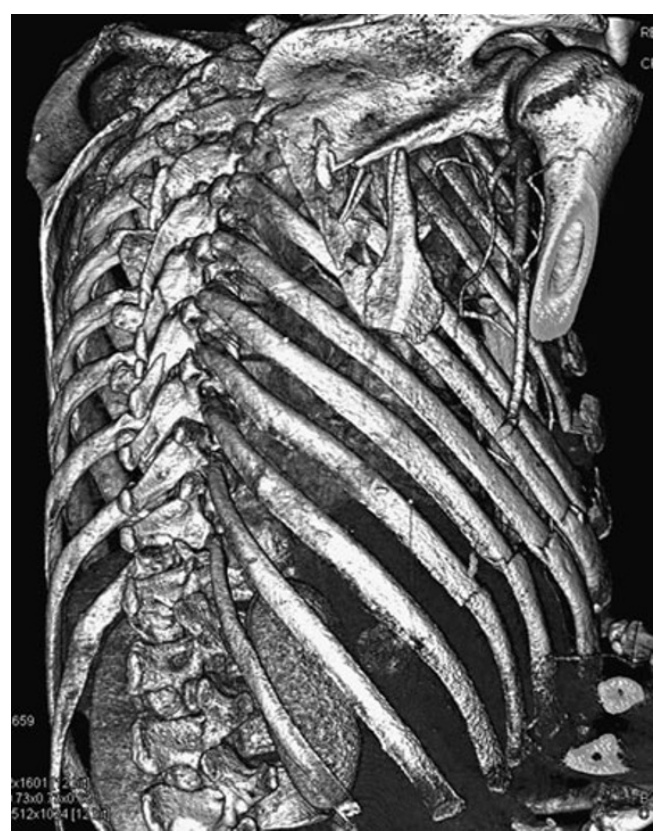

Fig. 8 Synoptic view of the chest by using a volume-rendered image. Smash fracture of the right scapula and multiple rib fractures on the right

of sternoclavicular dislocation, which is caused most commonly by indirect mechanisms. Anterior dislocation is more common and clinically less severe. Posterior dislocations may be associated with major vascular traumas [27].

\section{Thoracic spine}

Thoracic spine fractures represent $16-30 \%$ of all spinal injuries. They are often difficult to detect using the X-ray

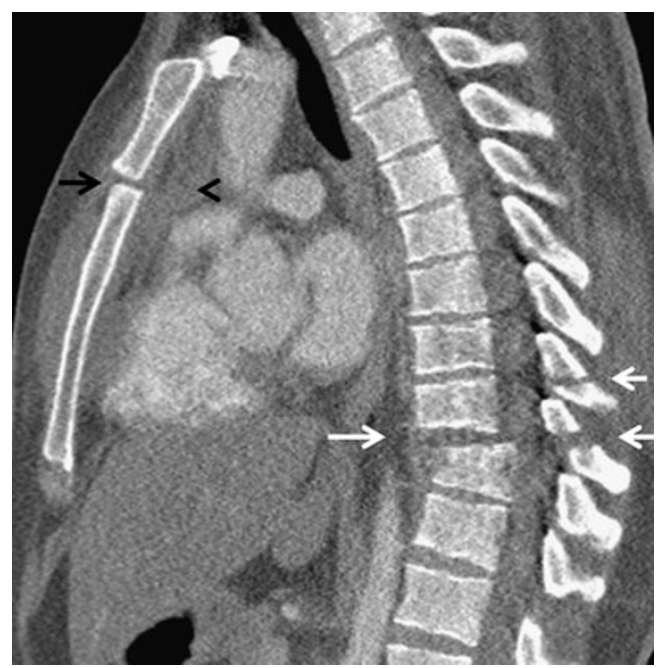

Fig. 9 Unstable dislocated flexion-distraction fracture, type $\mathrm{C} 1$ according to the AO classification (white arrows), combined with dislocation in the sternal angle (black arrow) and retrosternal hematoma (black arrowhead) examination because of superposition of other structures of the chest. The most common mechanism of injury includes flexion and axial forces. Rotating mechanisms are limited by the ribcage. Dislocations and unstable fractures, which occur in up to $50 \%$ of cases associated with a neurological deficit, are relatively common in this area. Fractures are categorized into two groups: minor and major. Minor fractures (spinous, transverse and articular processes, interarticular part) alone are rarely associated with spinal instability or neurological deficits. According to the AO classification, major fractures are divided into (A) compression fractures, (B) distraction fractures and (C) multidirection injury with shear and/or translation. Assessment of fracture stability is crucial in deciding on the therapeutic approach. Unstable fractures are those that can lead to increasing deformity or increasing neurological deficits, and require surgical stabilisation. According to Denis's three-column theory, fractures involving the middle column or two or more columns are regarded as unstable. CT is the first choice method for spinal fractures. Like in sternal fractures, sagittal reformations are very beneficial in this area (Fig. 9) $[3,28,29]$.

\section{Extrapleural hematoma}

When extrapleural hematoma is caused by injury of the intercostal artery, it is a potentially life-threatening condition [30]. It occurs relatively rarely as a result of the chest wall injury or a complication of interventions (drainage, insertion of the central venous catheter) [31-33]. Blood accumulates between the parietal pleura and endothoracal fascia. The Xray findings are often not specific. In the MDCT examination we can find blood collection separated from the lungs and pleural cavity by a thin strip of fat (Fig. 10) [33]. Larger hematomas have a biconvex shape [32]. Active bleeding from the intercostal artery may be seen in contrast material extravasation (Fig. 6).

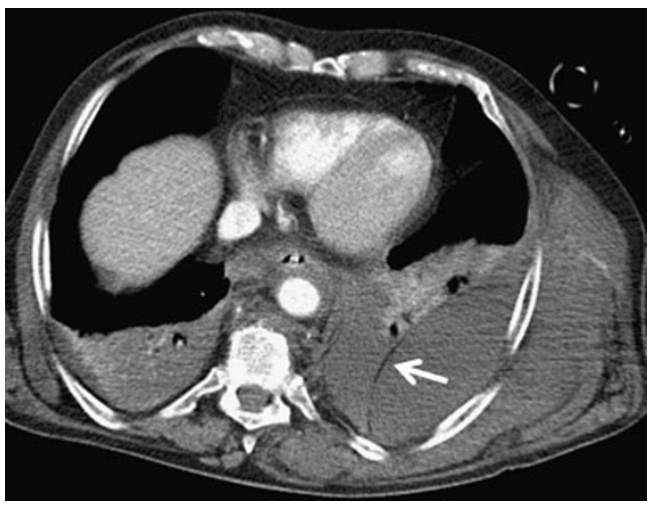

Fig. 10 Combination of extrapleural hematoma and hemothorax. Lenticular-shaped extrapleural hematoma is surrounded by a thin strip of fat (arrow) 


\section{Injuries of the pleura}

Pneumothorax

Pneumothorax frequently accompanies blunt and penetrating chest injuries. In cases of blunt trauma, it is the second most common finding after rib fractures. It is caused not only by direct injury to the pleura, but also by a rupture of the alveoli or bronchi accompanied by increased pressure in the airways. It can also be a complication of medical procedures. On CT it is manifested as a collection of gas in the pleural cavity accumulating behind the ventral thoracic wall. In chest Xray examinations performed in the supine position, it is often manifested very discretely, and in 30-55 \% of cases it cannot be seen at all (Fig. 11) [34, 35]. The clinical significance of pneumothorax depends not only on its size at the time of initial examination, but also on its development over time and on the overall condition of the patient. In a ventilated patient, a small overlooked pneumothorax can grow rapidly and cause a hemodynamic and ventilation instability [35]. Tension pneumothorax is a serious, life-threatening condition requiring immediate drainage. It results in an increase of intrathoracic
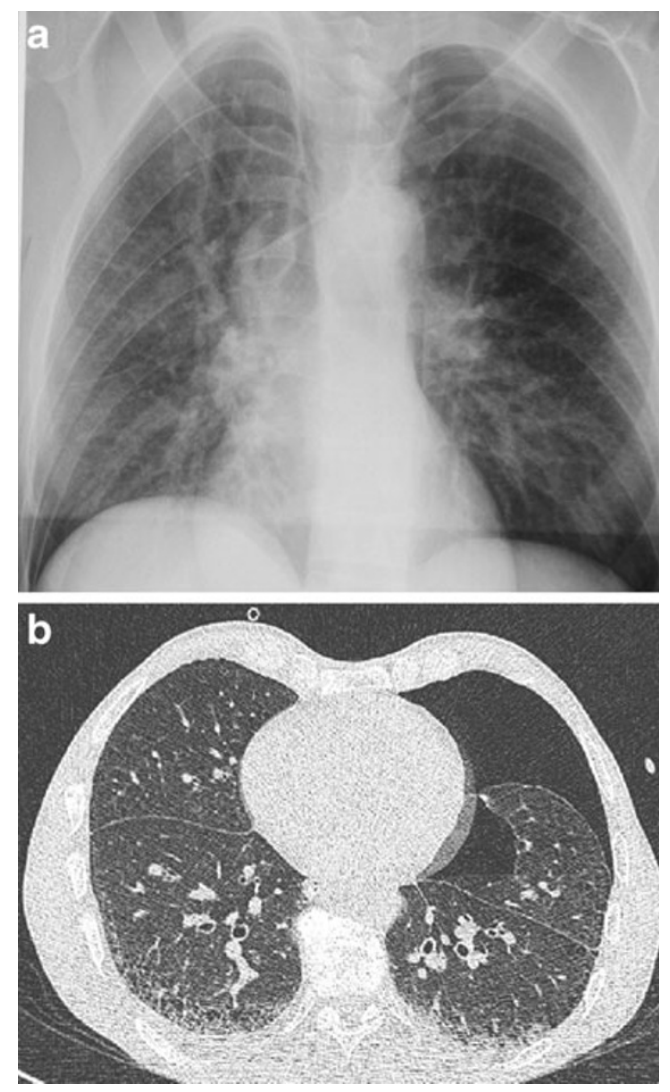

Fig. 11 a, b Pneumothorax: discrepancy between the supine chest radiograph and MDCT. a Chest radiograph reveals slightly increased transparency of the left lung and thin linear translucency contouring the left border of the lower mediastinum. b MDCT shows significant pneumothorax in the ventral part of the left pleural cavity pressure on the affected side with a subsequent compression of the mediastinal structures and reduction of cardiac diastolic filling [36]. It is often clinically diagnosed before MDCT. Its basic symptoms include an increase of the volume of the affected hemithorax, shift of the mediastinum to the healthy side, and compression of the mediastinal vessels (mainly veins), heart and depression of the diaphragmatic arch (Fig. 12) [16].

\section{Hemothorax}

Bleeding into the pleural cavity is most commonly caused by laceration of the pulmonary parenchyma and pleural injury. In these cases, bleeding is usually slowly progressive and limited. In case of injury to an artery (usually intercostal), bleeding progresses rapidly and requires surgical therapy. Less extensive bleeding does not have to be detected on the X-ray examination performed in the supine position. MDCT is an unrivalled method for the diagnosis of pleural fluid. Blood has a
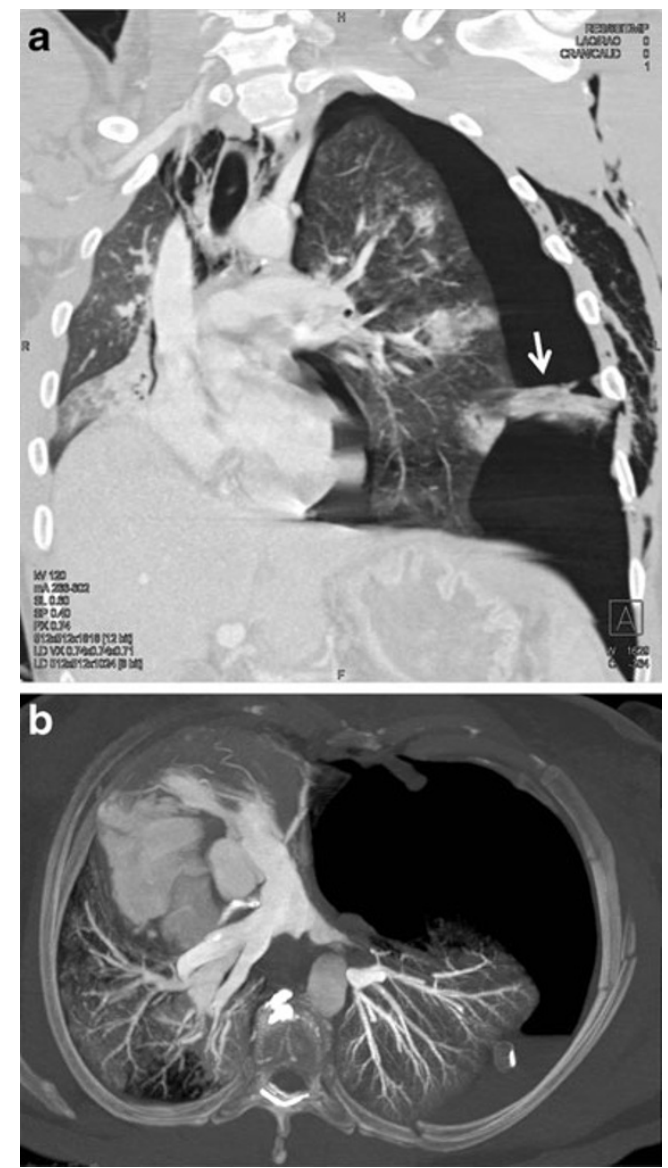

Fig. 12 a, b Tension pneumothorax, indication for immediate chest drainage. a Increased volume of the left hemithorax, multiple lung contusions, and capture of the lung parenchyma among the fragments of the ribs (arrow). b Maximum intensity projection: mediastinal shift to the right, dislocation and compression of the heart, and compression of the left pumonary artery 


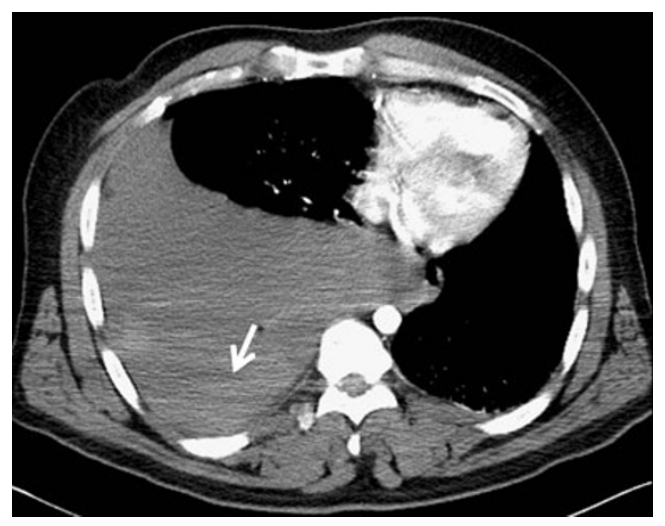

Fig. 13 Large layered hemothorax. Hyperdense clots $(50 \mathrm{HU})$ in the dorsal part of the right pleural cavity (arrow). Mediastinal shift to the left side, compression of the right atrium

higher density than water. Its value depends on the degree of coagulation. The density of liquid blood is between 30 and $50 \mathrm{HU}$, and the density of blood clots is $50-90 \mathrm{HU}$. Hematomas can sometimes have a layered structure (so-called hematocrit sign) (Fig. 13). In case of active bleeding from the injured artery, we can demonstrate leakage of the contrast substance into the hematoma $[3,16]$

\section{Lung parenchymal injuries}

Injury to the lung parenchyma includes pulmonary contusion, laceration, torsion and herniation. These conditions can be complicated by atelectasis, aspiration pneumonia or acute respiratory distress syndrome (ARDS) [9]. Lung trau$\mathrm{ma}$ is seldom a reason for surgical treatment. This is indicated in cases of injury of the major blood vessels, signs of active bleeding, large hematomas or hemodynamic instability in the context of pulmonary involvement.

\section{Pulmonary contusion}

Pulmonary contusion is the most common trauma of the lung parenchyma with an incidence between $30 \%$ and $70 \%$. It leads to hemorrhage, edema and secondary inflammatory infiltration of the pulmonary interstitium and alveoli [16]. It can be detected on the X-ray image with up to a 6$\mathrm{h}$ delay. On the other hand, CT is very sensitive to contusion changes, and it can detect them earlier and more accurately and allows their quantification, which is important in the determination of further treatment. In contusions exceeding $20-30 \%$ of the total lung volume, mechanical ventilation is indicated to prevent the development of complications, especially acute respiratory distress syndrome $[37,38]$. The
Fig. 14 Lung contusion volumetry using semiautomatic software volume (Siemens, Forchheim, Germany). Highlighted contusions occupy $26 \%$ of the total lung volume. The finding indicated artificial ventilation

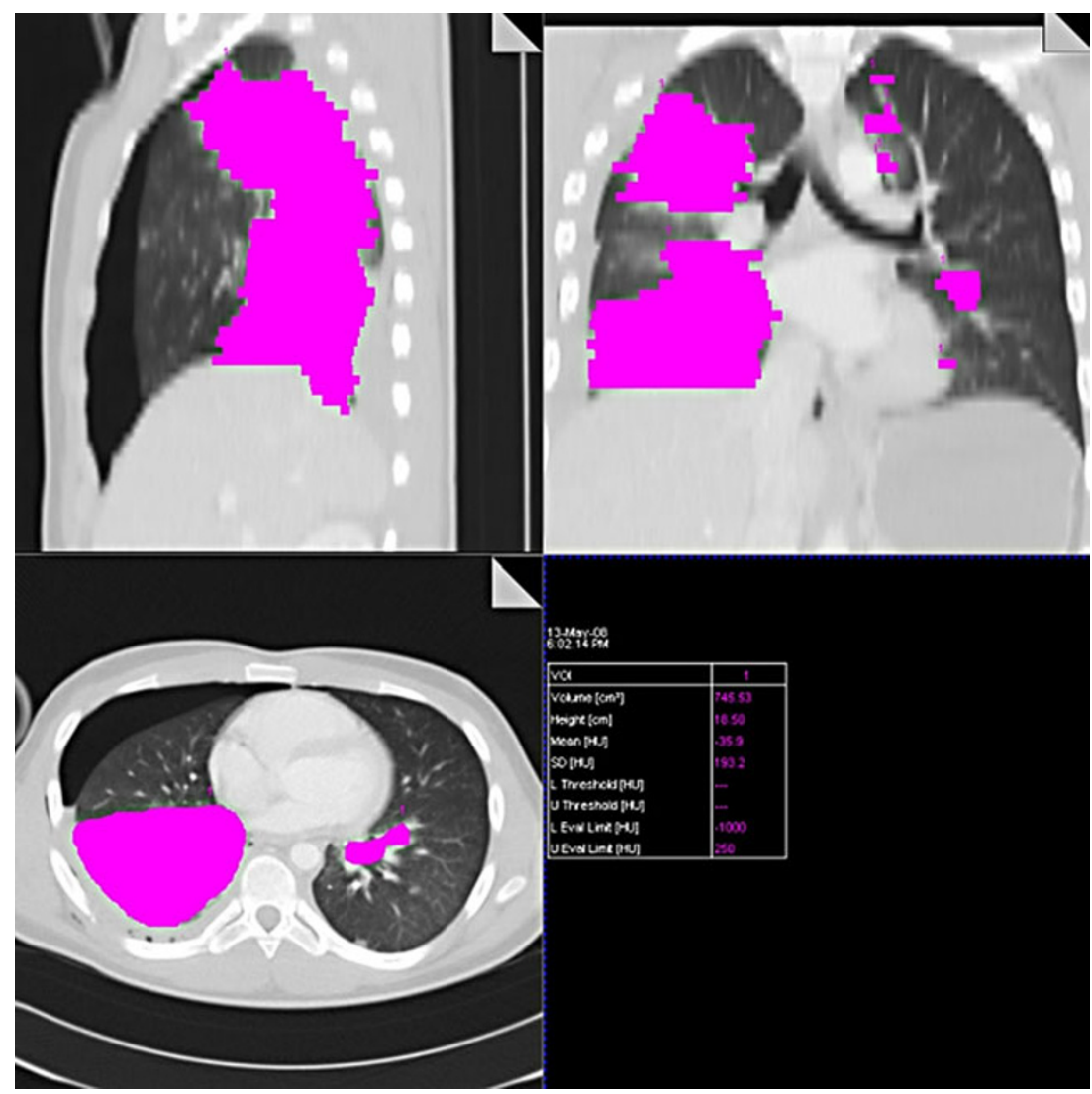




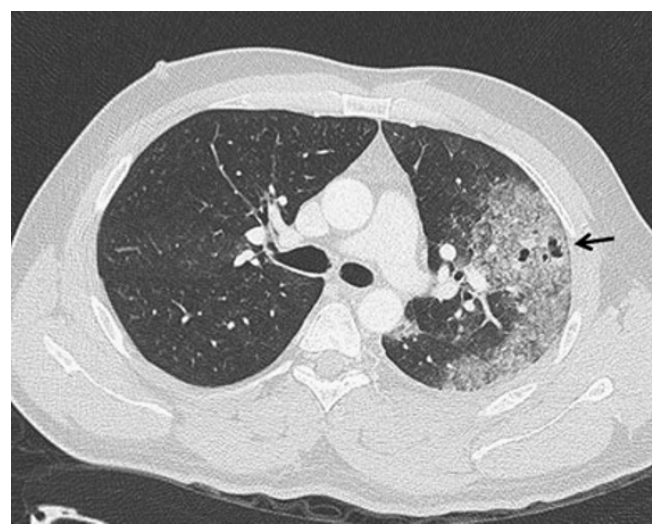

Fig. 15 Lung contusion with small lacerations (arrow), typical peripheral localisation

volume of the affected parenchyma can be estimated (each segment represents about $5 \%$ of the total lung volume) or exactly measured using dedicated volumetry software. The second option is more time-consuming (Fig. 14). If the CT scan is performed immediately after the trauma, further progression of the findings must be taken into account. Contusion achieves its maximum in the $24-48 \mathrm{~h}$ followed by absorption, which takes $1-2$ weeks $[3,5]$. The CT findings depend on the severity of parenchymal affection. In cases of interstitial involvement, areas of ground-glass opacity occur. More serious affection is accompanied by inhomogeneous alveolar condensation. Simultaneously, parenchymal lacerations can occur (Fig. 15). The peripheral areas of the lung are affected, and the distribution does not respect boundaries of the lobes and segments [9]. Differentiation of the contusion is difficult in the field of atelectasis, compression of the parenchyma with hemothorax or aspiration pneumonia (Fig. 16). The dependent density (the sickle-shaped parenchymal

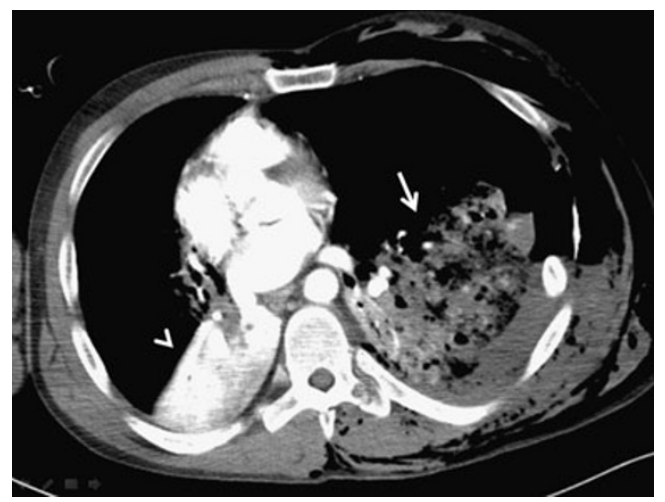

Fig. 16 Comparison between contusion and atelectasis. Contusion (arrow) is an inhomogenous, ill-demarcated, relatively hypodense parenchymal condensation with preservation of air in the bronchi (air bronchogram). Atelectasis (arrowhead) appears like homogenously enhancing well-demarcated parenchymal condensation without an air bronchogram

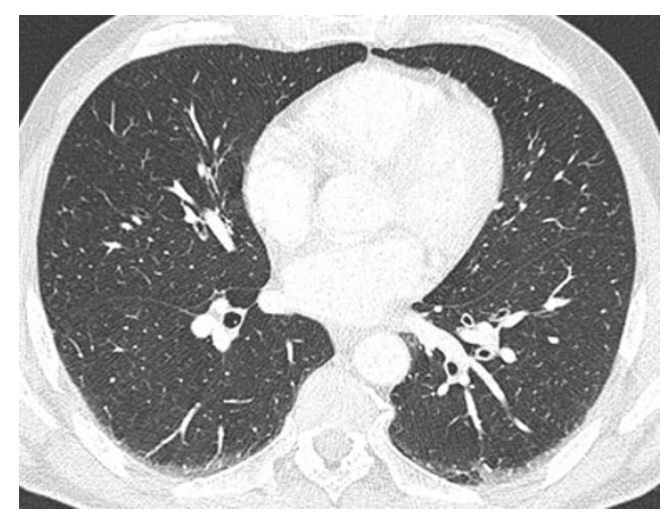

Fig. 17 Dependent density: symetrical bilateral sickle-like condensations in dependent parts of both lungs

condensations in the dependent lung regions) should not be considered as contusions (Fig. 17) [39].

Lung laceration

Laceration occurs in penetrating injuries as a result of direct tearing of the parenchyma in blunt traumas as a result of
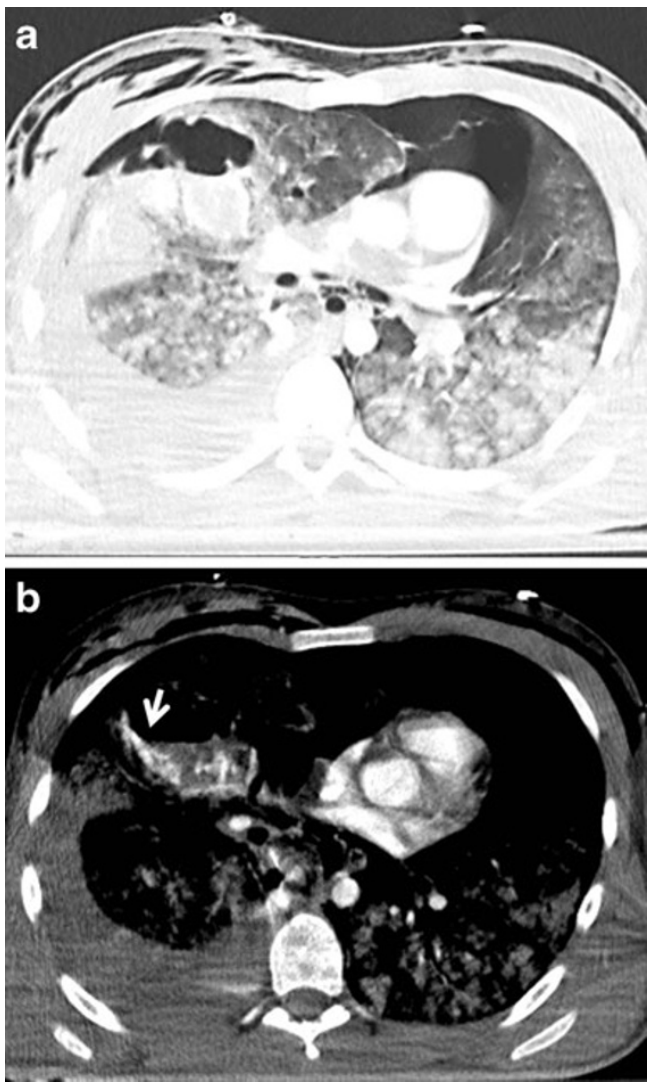

Fig. 18 a, b Extensive lung laceration, large bilateral contusions. a Cavities with air-fluid levels in the right upper lobe, right-sided pneumothorax and fluidothorax, bilateral subcutaneous emphysema, pneumomediastinum, mediastinal shift to the left. b Extravasation of the contrast material into the cavity due to active bleeding; the finding was an indication for surgery 
shearing forces. Laceration was considered a rare finding before the era of CT. At present we can find it relatively commonly because of the broad use of $\mathrm{CT}$ in traumatised patients. X-ray examination is usually negative because of superposition of simultaneously occurring contusion changes. If a laceration reaches the pleural cavity, it can cause pneumothorax or hemothorax. Laceration closed in the parenchyma has the appearance of a thin-walled, spherical, oval or tubular cavity containing air, blood or their combination (Fig. 18). There are usually contusion changes around it. When they are absorbed the laceration, which does not contain air, can mimic a pulmonary node $[9,16]$.

\section{Lung torsion}

Lung torsion is a rare, life-threatening complication of pulmonary surgery or trauma. In rare cases it can occur spontaneously. It affects the whole lung or a lobe [40]. On CT the torsion is manifested as an abnormal position of the lung or its lobe with condensation and obstruction of the airways or
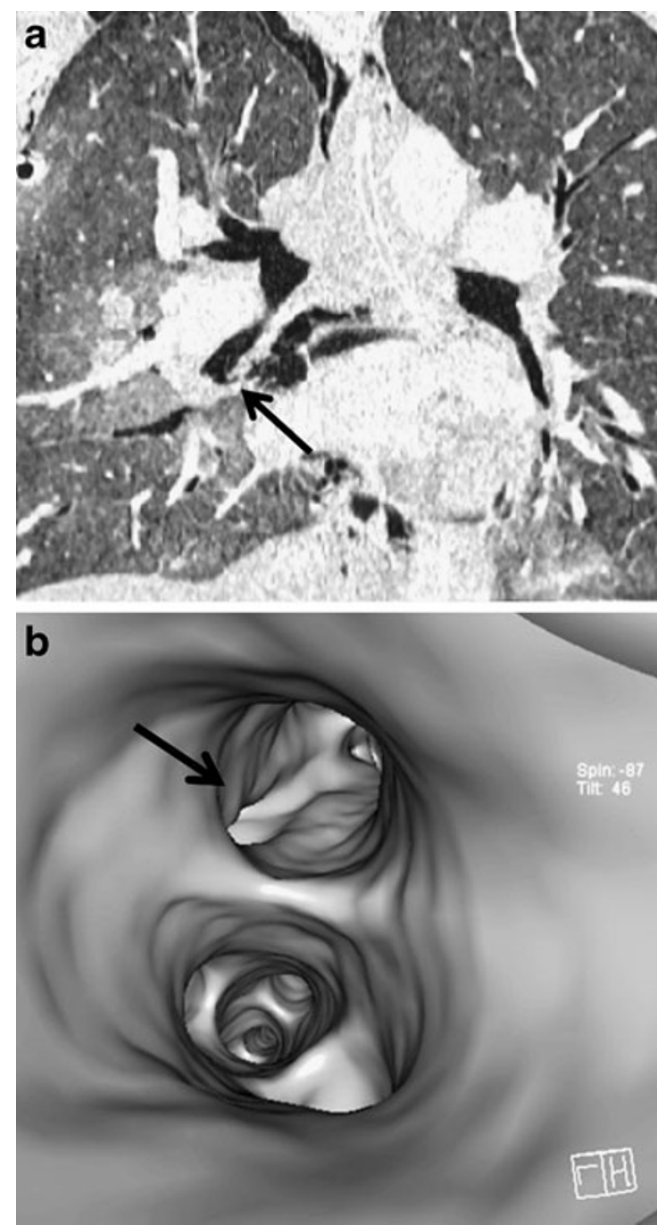

Fig. 19 a, b Tiny tear of the right middle lobar bronchus, contribution of virtual bronchoscopy. a Multiplanar reformation - the tear is poorly visible (arrow). b Virtual bronchoscopy: clear visualization of the tear (arrow) distortion and abnormal position of the bronchi and blood vessels [41].

\section{Lung herniation}

Like torsion, lung herniation is a rare complication of chest injuries. It occurs at the site of an inherited or acquired defect of the chest wall with a significant increase in intrathoracic pressure. The acquired chest wall defects can be caused by multiple fractures of the ribs or by sterno- and costoclavicular dislocation. If parenchymal tearing occurs at the same time, the herniation may be associated with pneumothorax and hemothorax [16, 42].

\section{Injuries of trachea and bronchi}

Laceration of the trachea and bronchi is caused by two mechanisms - by increasing the intraluminal pressure with a closed vocal slot due to a sudden compression of the chest or by compression of the main bronchi against the spine. The neck part of the trachea can also be injured by a direct impact. The most common location of lacerations in the

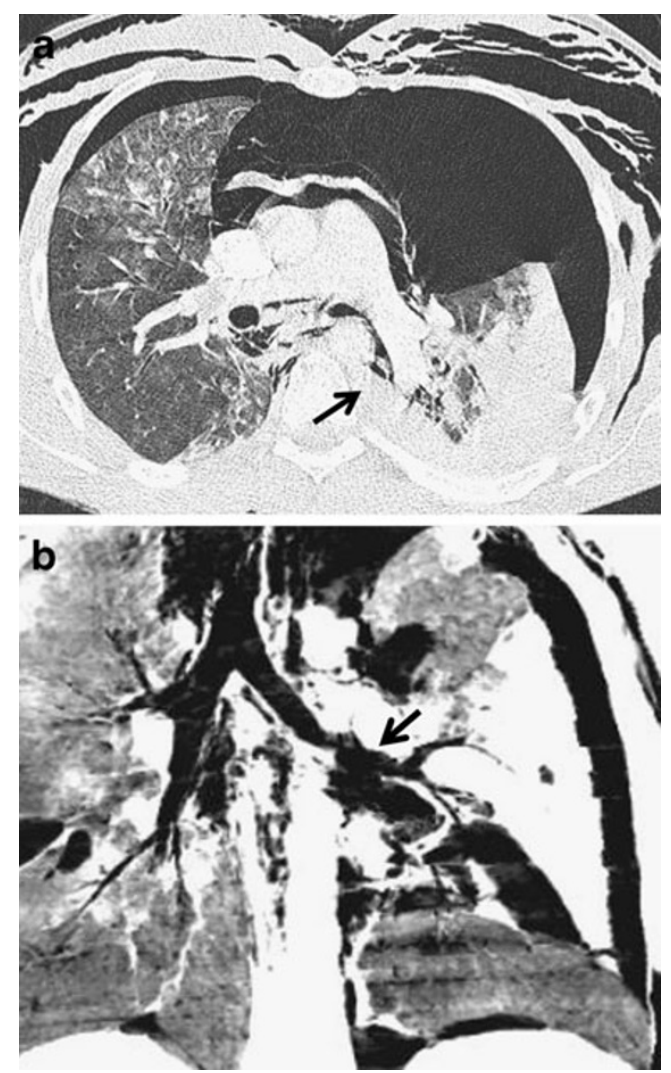

Fig. 20 a, b Rupture of the left main bronchus (arrow). a Discontinuity of the bronchus, fallen lung sign, pneumomediastinum, bilateral pneumothorax and subcutaneous emphysema. b Coronal minimum intensity projection shows leakage of air through a tear 
trachea is $2 \mathrm{~cm}$ above the carina and in the main bronchi (more in the right) at a distance of $2.5 \mathrm{~cm}$ from the carina [3, 22]. A tracheobronchial trauma is often difficult to find. In addition to thin slices in the axial plane and multiplanar reformations, the $3 \mathrm{D}$ visualisation of the bronchial tree (using virtual bronchoscopy or the volume-rendering technique) can be helpful for detection of the rupture (Fig. 19) [43]. A defect in the wall of the airways with leakage of air into the surrounding area is a direct sign of the airway trauma. There are other relatively specific findings such as a collapsed lung located in the most dependent part of the hemithorax (fallen lung sign) and persistence of pneumothorax following the insertion of the intrathoracic drain (Fig. 20). Other symptoms, such as pneumomediastinum, subcutaneous emphysema in the neck and chest, pneumothorax and hemothorax, are nonspecific and more commonly have other causes [44].

\section{Esophageal rupture}

Rupture of the esophagus as a part of a blunt or penetrating chest trauma is rare. Most often, it occurs in the cervical and upper mediastinal part. It is usually caused by bone fragments from the injury of adjacent sections of the spine. The CT findings include thickening of the esophageal wall, pneumomediastinum and paraesophageal fluid collections and fluidothorax (more often in the left) $[45,46]$. If an oral contrast agent is administered before $\mathrm{CT}$, its leakage through the esophageal wall defect can be demonstrated [22].

\section{Diaphragmatic rupture}

Diaphragmatic rupture occurs in $0.8-7 \%$ of patients hospitalised with a blunt trauma [47]. It is a frequently overlooked injury but it is clinically very serious. It occurs more often on the left (2/3-3/4 of cases), probably because the right half of the diaphragm is protected by the liver. Laceration in the right side is more difficult to detect. Sensitivity of the initial X-ray examination is low, $17 \%$ and $27-60 \%$ on the right and left side, respectively [48]. Compared to this, MDCT reaches a sensitivity of 50-83\% and $78-100 \%$ in the right and left side, respectively. Rarely, herniation to the pericardium or through the hiatus esophagus can occur [8]. Evaluation of multiplanar reformations in
Fig. 21 a, b, c Signs of rupture of the diaphragm. a Herniation of abdominal organs into the left hemithorax (arrow), clubshaped edge of the ruptured diaphragm (arrowhead). b Contact of herniated viscera with the most dependent part of the chest wall: dependent viscera sign (arrow). c Strangulation of herniated viscera in the defect of the diaphragm: collar sign (arrow)
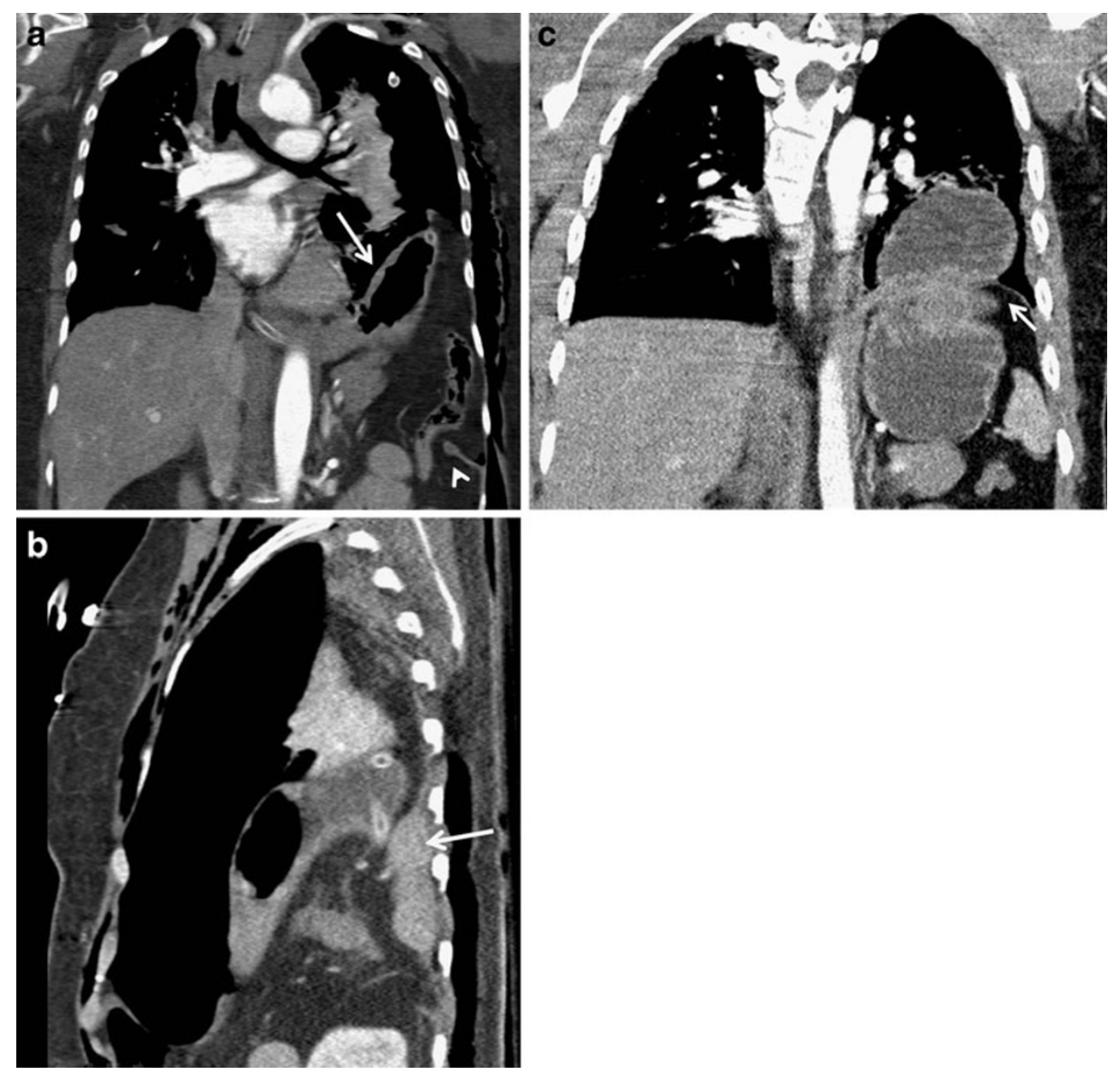
the coronal and sagittal plane is very important for making the diagnosis [49-53]. Diaphragmatic rupture is associated with a herniation of the abdominal organs, which can arise at the time of injury or later. The CT signs of a diaphragmatic rupture include discontinuity of the diaphragm with thickening at the edge of the defect, herniation of the abdominal organs into the chest, strangulation of the organs at their passage through the diaphragmatic defect (collar sign) and location of herniated abdominal organs in the dorsal part of the thorax where they are in contact with the chest wall (dependent viscera sign) (Fig. 21) [50]. Diaphragmatic injuries are often associated with injuries of the spleen, liver, lungs and rib fractures [54].

\section{Trauma of large vessels}

Trauma of the large chest vessels represents about $5 \%$ of all vascular traumas, but its incidence is probably underestimated. Although relatively infrequent, it is the second most

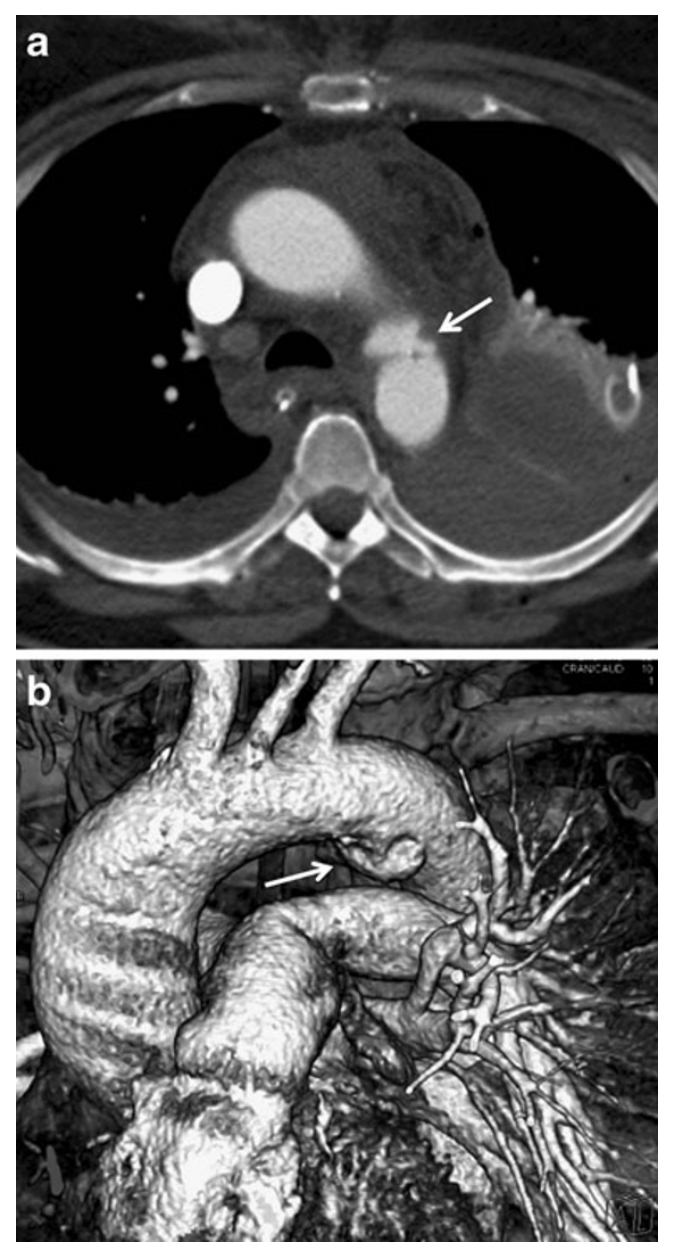

Fig. 22 a, b Aortic isthmus laceration. a Pseudoaneurysm in a typical location (arrow), mediastinal hematoma, bilateral hemothorax. b Volume-rendered image: 3D view of the pseudoaneurysm (arrow) common cause of death after head injuries [55, 56]. The aorta is the most commonly affected vessel. Rarely, we can see injuries of the aortic branches, internal thoracic artery or major mediastinal veins. In the case of a blunt trauma, there are three traumatic mechanisms leading to the trauma-deceleration, torsion and increased intraluminal pressure. Findings on the $\mathrm{X}$-ray examination are usually nonspecific and do not allow to differentiate trauma of the large vessels from other less serious conditions with similar X-ray findings, as described above. [5, 12]. If this type of trauma is suspected, MDCT is currently the first-choice method. Its sensitivity and specificity are almost $100 \%$. Angiography is indicated only in cases of doubt and for navigation of the interventional procedures [57].

\section{Aortic trauma}

Mortality of untreated aortic trauma exceeds $95 \%$ [58]; 80$90 \%$ of patients die at the place of accident. Those who come to the hospital have a $70 \%$ chance of survival if the trauma is quickly diagnosed and treated $[59,60]$. Treatment is either surgical or radiointerventional (endovascular stent grafting). The most common site of injury (approximately $90 \%$ ) is the isthmus, which represents the transition between the relatively mobile arch and fixed descending part. Other less frequent localisations of trauma include the ascending aorta above the aortic valve, branching of the truncus brachiocephalicus and the distal segment of the descending aorta.
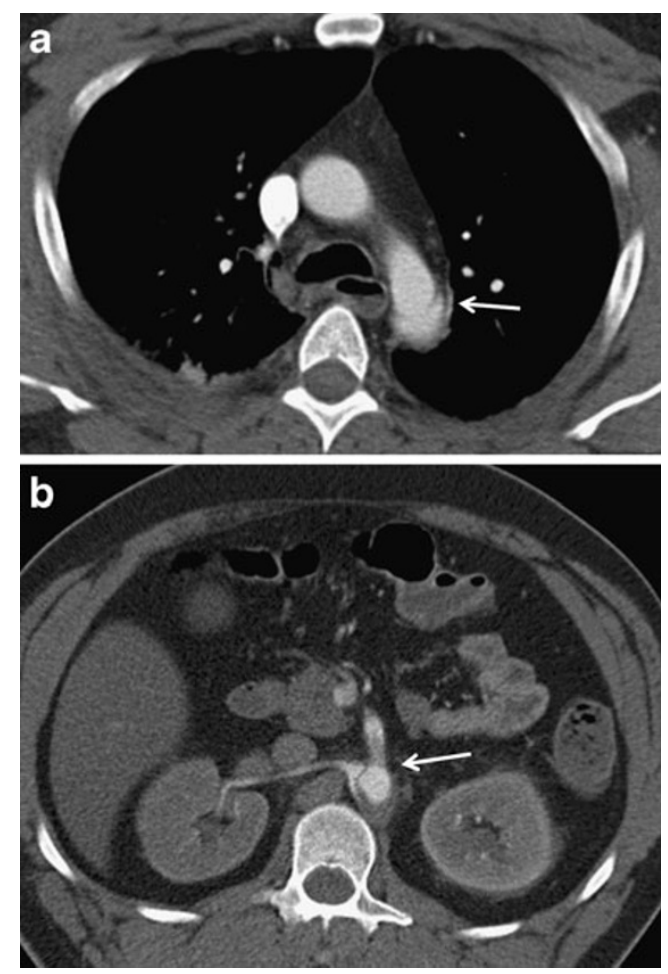

Fig. 23 a, b Traumatic dissection of the descending aorta. a Proximal border at the level of the isthmus. $\mathbf{b}$ Involvement of the abdominal part 
Fig. 24 a, b, c Pitfalls in diagnostics of aortic trauma. a Motion artifact mimicking a tear of the ascending aorta. $\mathbf{b}$ Ductus diverticulum: smooth shape, no mediastinal hematoma, connection to the falciform ligament (arrow). c Supreme intercostal vein (arrow). Note the different density after correcting the window setting (arrowhead)
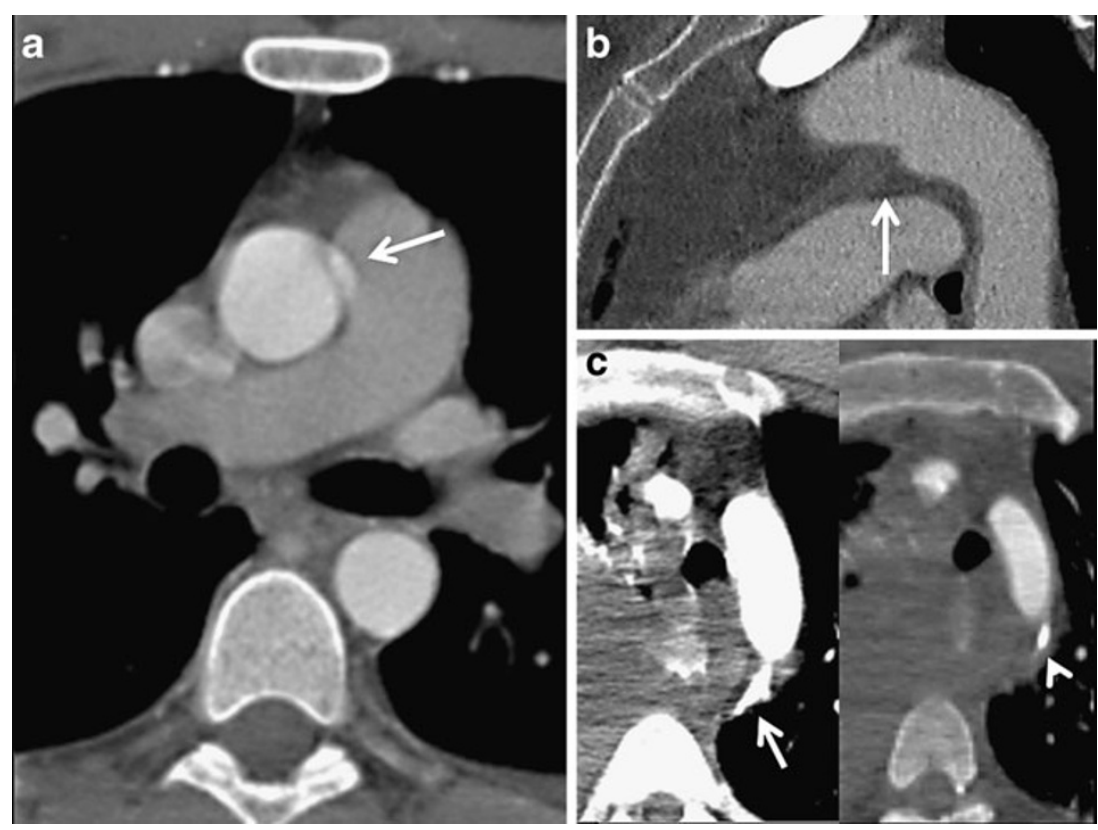

Impairment of the wall spreads from the intima to adventitia $[3,61,62]$. In patients who come to the hospital, we can more often find partial lesions. A complete wall rupture (transsection) usually results in immediate death. Direct signs of aortic trauma are pseudoaneurysm (Fig. 22), direct extravasation of contrast medium into the mediastinum, intimal flap (Fig. 23), irregular contour, mural thrombus or pseudocoarctation $[5,12]$. Indirect and less specific signs of aortic trauma include periaortic hematoma, hemothorax and hemopericardium. Periaortic hematoma may come from small blood vessels in the affected area or from the vasa vasorum and may not be a sign of aortic wall injury. Problems with interpretation of findings may arise in case of ulcerated atherosclerotic plaques or congenital anomalies such as the ductus diverticulum or supreme intercostals vein. In these cases, the aorta is externally well demarcated and without periaortic hematoma. The edge of the ductus diverticulum is smooth and regular [63]. In some cases we can identify the ligamentum arteriosum,

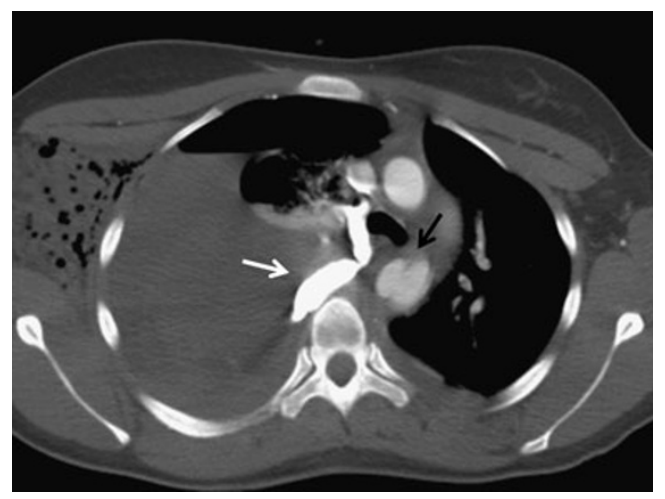

Fig. 25 Rupture of the azygos vein with contrast material leakage into the large hemothorax (white arrow) combined with aortic pseudoaneurysm (black arrow) which is linked to it. The supreme intercostal vein flows into the brachiocephalic vein and may run along the left side of the aortic arch. On the ascending aorta above the valve, motion artifacts can often be seen (Fig. 24). In case of diagnostic uncertainty, it is possible to add a targeted examination with ECG synchronisation.

\section{Injuries of other large vessels}

Injuries of other blood vessels, such as the internal thoracic artery, branches of the aortic arch, pulmonary artery or mediastinal veins, are less common than aortic trauma, but their severity is similar. In case of bleeding from the internal thoracic artery, a hematoma occurs in the anterior mediastinum, and it may compress the right ventricle [64]. Injury of the branches of the aortic arch occurs most frequently in the traction mechanism in case of abnormal extension of the

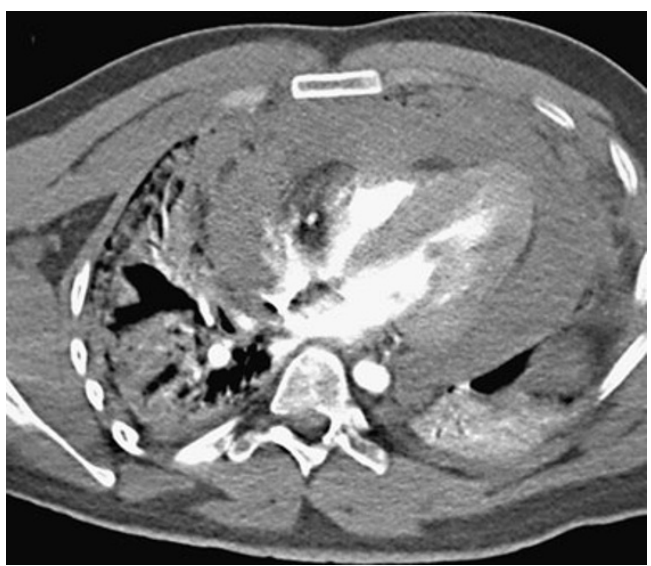

Fig. 26 Hemopericardium with heart tamponade: compression of all heart parts 
neck or pulling of the arm. It is manifested by occlusion, dissection of the affected artery or extravasation of contrast material into the hematoma, which is typically located in the upper part of the mediastinum. Involvement of the common carotid artery can cause stroke [8]. Rare injuries of the venous structures are manifested as hematoma with extravasation of contrast medium (Fig. 25). The mechanism is similar to injuries of the arteries. Injury of the vena cava superior can spread to the right atrium as well $[65,66]$.

\section{Heart and pericardium injuries}

Contusion is the most common heart injury affecting usually the right ventricle. It is generally radiologically silent and causes arrhythmia or other electrocardiographic changes. Because of its very high mortality, cardiac rupture is rarely diagnosed by imaging methods [67]. It can be manifested as a hemopericardium and extravasation of contrast material from the injured cardiac cavity [68]. Rarely, due to a coronary artery injury, myocardial infarction can occur [69]. Diagnosis of hemopericardium using X-ray examination is extremely problematic. The finding can be negative even in cases of cardiac tamponade. In contrast, the CT examination as well as ultrasound is a highly sensitive method. Cardiac tamponade is manifested by flattening and a reduction of the cardiac parts (Fig. 26) [70]. More rarely, we can see pneumopericardium, which, if large, may also result in cardiac tamponade [71]. Herniation of the heart is a serious complication of traumatic rupture of the pericardium [72].

\section{Conclusion}

Imaging methods are an integral part of the diagnostic algorithm in chest injuries. MDCT is its main component. It shows traumatic changes quickly, accurately and clearly, and allows their classification. Therefore, a traumatic radiologist becomes a significant member of the team making decisions about the therapeutic process.

Open Access This article is distributed under the terms of the Creative Commons Attribution License which permits any use, distribution, and reproduction in any medium, provided the original author(s) and the source are credited.

\section{References}

1. Wintermark M, Schnyder P (2004) MDCT of chest trauma. In: Schoepf UJ (ed) Multidetector-row CT of the thorax. Springer, Berlin, Heidelberg, New York, pp 409-421
2. LoCicero J, Mattox KL (1989) Epidemiology of chest trauma. Surg Clin North Am 69:15-19

3. Lomoschitz FM, Eisenhuber E, Linnau KF, Peloschek P, Schoder M, Bankier AA (2003) Imaging of chest trauma: radiological patterns of injury and diagnostic algorithms. Eur J Radiol 48:61-70

4. Scaglione M, Pinto A, Pedrosa I, Sparano A, Romano L (2008) Multi-detector row computed tomography and blunt chest trauma. European J Radiol 65:377-388

5. Mirvis SE (2005) Imaging of acute thoracic injury: the advent of MDCT screening. Semin Ultrasound CT MRI 26:305-331

6. O'Connor JV, Adamski J (2010) The diagnosis and treatment of non-cardiac thoracic trauma. J R Army Med Corps 156:514

7. Trupka A, Waydas C, Hallfeldt KK, Nast-kolb D, Pfeifer KJ, Schweiberer L (1997) Value of thoracic computed tomography in the first assessment of severely injured patients with blunt chest trauma: results of a prospective study. J Trauma 43:405412

8. Kaewlai R, Avery LL, Asrani AV, Novelline RA (2008) Multidetector CT of blunt thoracic trauma. RadioGraphics 28:1555-1570

9. Elmali M, Baydin A, Nural MS, Arslan B, Ceyhan M, Gürmen N (2007) Lung parenchymal injury and its frequency in blunt thoracic trauma: the diagnostic value of chest radiography and thoracic CT. Diagn Interv Radiol 13:179-182

10. Brink M, Deunk J, Dekker HM et al (2008) Added value of routine chest MDCT after blunt trauma: evaluation of additional findings and impact on patient management. Am J Roentgenol 190:1591-1598

11. Brink M, Kool DR, Dekker HM et al (2009) Predictors of abnormal chest $\mathrm{CT}$ after blunt trauma: a critical appraisal of the literature. Clin Radiol 64:272-283

12. Mirvis SE, Shanmuganathan K, Buell J et al (1998) Use of spiral computed tomography for the assessment of blunt trauma patients with potential aortic injury. J Trauma 45:922-930

13. Kaiser M, Whealon M, Barrios C et al (2010) The clinical significance of occult thoracic injury in blunt trauma patients. Am Surg 76:1063-1066

14. Hauser CJ, Visvikis G, Hinrichs C et al (2003) Prospective validation of computed tomographic screening of the thoracolumbar spine in trauma. J Trauma 55:228-234

15. Guerrero-Lopez F, Vazquez-Mata G, Cazar-Romero PP, FernandezMondejar E, Guayo-Hoyos E, Linde-Valverde CM (2000) Evaluation of the utility of computed tomography in the initial assessment of the critical care patient with chest trauma. Crit Care Med 28:1370 1375

16. Sangster GP, González-Beicos A, Carbo AI et al (2007) Blunt traumatic injuries of the lung parenchyma, pleura, thoracic wall, and intrathoracic airways: multidetector computer tomography imaging findings. Emerg Radiol 14:297-310

17. Mullinix AJ, Foley WD (2004) Multidetector computed tomography and blunt thoracoabdominal trauma. J Comput Assist Tomogr 28:S20-S27

18. Vergnion M, Lambert JL (2006) A protocol of trauma care in the emergency service including MDCT imaging. Acta Anaesthesiol Belg 57:249-252

19. Moore MA, Wallace EC, Westra SJ (2009) The imaging of paediatric thoracic trauma. Pediatr Radiol 39:485-496

20. Tack D (2010) Radiation dose optimization in thoracic imaging. JBR-BTR 93:15-19

21. Schertler T, Glücker T, Wildermuth S, Jungius KP, Marincek B, Boehm T (2005) Comparison of retrospectively ECG-gated and nongated MDCT of the chest in an emergency setting regarding workflow, image quality, and diagnostic certainty. Emerg Radiol 12:19-29

22. Beigelman-Aubry C, Baleato S, Le Guen M, Brun AL, Grenier P (2008) Chest trauma: spectrum of lesions. J Radiol 89:1797-1811

23. Ferda J, Mirka H, Baxa J, Kreuzberg B (2009) Multidetector computed tomography. Galen, Prague, pp 116-117, 210-211 
24. Haapamaki VV, Kiuru MJ, Koskinen SK (2004) Multidetector CT in shoulder fractures. Emerg Radiol 11:89-94

25. McGinnis M, Denton JR (1989) Fractures of the scapula: a retrospective study of 40 fractured scapulae. J Trauma 29:14881493

26. Kim EY, Yang HJ, Sung YM, Hwang KH, Kim JH, Kim HS (2011) Sternal fracture in the emergency department: diagnostic value of multidetector $\mathrm{CT}$ with sagittal and coronal reconstruction images. Eur J Radiol 14 [Epub ahead of print]

27. Maier D, Jaeger M, Izadpanah K, Bornebusch L, Südkamp NP (2011) Traumatic injuries of the sternoclavicular joint. Unfallchirurg 114:611-621

28. Denis F (1984) Spinal instability as defined by the three-column spine concept in acute spinal trauma. Clin Orthop Relat Res 189:65-76

29. Aebi M (2010) Classification of thoracolumbar fractures and dislocations. Eur Spine J 19:2-7

30. Igai H, Okumura N, Ohata K, Matsuoka T, Kameyama K, Nakagawa $\mathrm{T}$ (2008) Rapidly expanding extrapleural hematoma. Gen Thorac Cardiovasc Surg 56:515-517

31. Rashid MA, Wikström T, Örtenwall P (2000) Nomenclature, classification, and significance of traumatic extrapleural hematoma. J Trauma Inj Infect Crit Care 49:286-290

32. Chung JH, Carr RB, Stern EJ (2010) Extrapleural hematomas: imaging appearance, classification, and clinical significance. J Thorac Imaging 26:218-223

33. Aquino SL, Chiles C, Oaks T (1997) Displaced extrapleural fat as revealed by CT scanning: evidence of extrapleural hematoma. Am J Roentgenol 169:687-689

34. Ball CG, Kirkpatrick AW, Laupland KB et al (2005) Incidence, risk factors, and outcomes for occult pneumothoraces in victims of major trauma. J Trauma 59:917-924

35. Omar HR, Abdelmalak H, Mangar D, Rashad R, Helal E, Camporesi EM (2010) Occult pneumothorax, revisited. J Trauma Manag Outcome 4:3-6

36. Leigh-Smith S, Harris T (2005) Tension pneumothorax - time for a re-think? Emerg Med J 22:8-16

37. de Moya MA, Manolakaki D, Chang Y et al (2011) Blunt pulmonary contusion: admission computed tomography scan predicts mechanical ventilation. J Trauma 71:1543-1547

38. Strumwasser A, Chu E, Yeung L, Miraflor E, Sadjadi J, Victorino GP (2011) A novel CT volume index score correlates with outcomes in polytrauma patients with pulmonary contusion. J Surg Res 170:280-285

39. Webb WR, Naindich DP, Mueller NL (2001) High-resolution CT of the lung. Lippincott Willkiams \& Wilkins, Philadelphia, p 604

40. Wong PS, Goldstraw P (1992) Pulmonary torsion: a questionnaire survey and a survey of the literature. Ann Thorac Surg 54:286-288

41. Gilkeson RC, Lange P, Kirby TJ (2000) Lung torsion after lung transplantation evaluation with helical CT. Am J Roentgenol 174:1341-1343

42. Sukkarieh F, Vanmeerhaeghe A, Brasseur P (2002) Post traumatic intercostal lung herniation: a case report. J Radiol 83:10851087

43. Le Guen M, Beigelman C, Bouhemad B, Wenjïe Z, Marmion F, Rouby JJ (2007) Chest computed tomography with multiplanar reformatted images for diagnosing traumatic bronchial rupture: a case report. Crit Care 11:R94

44. Rezende-Neto JB, Hoffmann J, Al Mahroos M et al (2010) Occult pneumomediastinum in blunt chest trauma: clinical significance. Injury 41:40-43

45. Tjardes T, Wafaizadeh A, Steinhausen E, Krakamp B, Bouillon B (2009) Extension injury of the thoracic spine with rupture of the oesophagus and successful conservative therapy of concomitant mediastinitis. Eur Spine J 18:240-244

46. Young CA, Menias CO, Bhalla S, Prasad SR (2008) CT features of esophageal emergencies. RadioGraphics 28:1541-1553

47. Meyers BF, McCabe CJ (1993) Traumatic diaphragmatic hernia: occult marker of serious injury. Ann Surg 218:783-790

48. Gelman R, Mirvis SE, Gens D (1991) Diaphragmatic rupture due to blunt trauma: sensitivity of plain chest radiographs. Am J Roentgenol 156:51-57

49. Larici AR, Gotway MB, Litt HI et al (2002) Helical CT with sagittal and coronal reconstructions: accuracy for detection of diaphragmatic injury. Am J Roentgenol 179:451-457

50. Reesa O, Mirvis SE, Shanmuganathan K (2005) Multidetector-row $\mathrm{CT}$ of right hemidiaphragmatic rupture caused by blunt trauma: a review of 12 cases. Clin Radiol 60:1280-1289

51. Shanmuganathan K, Killeen K, Mirvis SE, White CS (2000) Imaging of diaphragmatic injuries. J Thorac Imaging 15:104-111

52. Killeen K, Mirvis SE, Shanmuganathan K (1999) Helical CT of diaphragmatic rupture caused by blunt trauma. Am J Roentgenol 173:1611-1616

53. Bhullar IS, Block EF (2011) CT with coronal reconstruction identifies previously missed smaller diaphragmatic injuries after blunt trauma. Am Surg 77:55-58

54. Al-Refaie RE, Awad E, Mokbel EM (2009) Blunt traumatic diaphragmatic rupture: a retrospective observational study of 46 patients. Interact Cardiovasc Thorac Surg 9:45-49

55. Mattox KL, Feliciano DV, Burch J, Beall AC Jr, Jordan GL Jr, De Bakey ME (1989) Five thousand seven hundred sixty cardiovascular injuries in 4459 patients. Epidemiologic evolution 1958 to 1987. Ann Surg 209:698-705

56. Onan B, Demirhan R, Öz K, Onan IS (2011) Cardiac and great vessel injuries after chest trauma: our 10-year experience. Turk J Trauma Emerg Surg 17:423-429

57. Steenburg SD, Ravenel JG (2008) Acute traumatic thoracic aortic injuries: experience with 64-MDCT. Am J Roentgenol 191:15641569

58. Parmley L, Thomas W, Manion W, Jahnke E (1958) Nonpenetrating traumatic injury of the aorta. Circulation 17:10861101

59. Holmes JH, Bloch RD, Hall RA, Carter YM, Karmy-Jones RC (2002) Natural history of traumatic rupture of the thoracic aorta managed nonoperatively: a longitudinal analysis. Ann Thorac Surg 73:1149-1154

60. von Oppell UO, Dunne TT, De Groot MK, Zilla P (1994) Traumatic aortic rupture: twenty-year metaanalysis of mortality and risk of paraplegia. Ann Thorac Surg 58:585-593

61. Kodali S, Jamieson WR, Leia-Stephens M, Miyagishima RT, Janusz MT, Tyers GF (1991) Traumatic rupture of the thoracic aorta. A 20-year review: 1969-1989. Circulation 84:4046

62. Feczko JD, Lynch L, Pless JE, Clark MA, McClain J, Hawley DA (1992) An autopsy case review of 142 nonpenetrating (blunt) injuries of the aorta. J Trauma 33:846-849

63. Fisher RG, Sanchez-Torres M, Whigham CJ, Thomas JW (1997) "Lumps" and "bumps" that mimic acute aortic and brachiocephalic vessel injury. Radiographics 17:825-834

64. Braatz T, Mirvis SE, Killeen K, Lightman NI (2001) CT diagnosis of internal mammary artery injury caused by blunt trauma. Clin Radiol 56:120-123

65. Chaumoitre K, Zappa M, Portier F, Panue M (1997) Rupture of the right atrium-superior vena cava junction from blunt thoracic trauma: helical CT diagnosis. Am J Roentgenol $169: 1753$ 
66. Holly BP, Steenburg SD (2011) Multidetector CT of blunt traumatic venous injuries in the chest, abdomen, and pelvis. Radiographics 31:1415-1424

67. Yu-Yun N, Ming-Shian L, Kuo-Sheng L et al (2009) Blunt traumatic cardiac rupture: therapeutic options and outcomes. Inj Int J Care Inj 40:938-945

68. Shanmuganathan K, Mirvis SE (1999) Imaging diagnosis of nonaortic thoracic injury. Radiol Clin North Am 37:533551

69. Malbranque G, Serfaty JM, Himbert D, Steg PG (2011) Myocardial infarction after blunt chest trauma: usefulness of cardiac ECG-gated
CT and MRI for positive and aetiologic diagnosis. Emerg Radiol $18: 271-274$

70. Huang YK, Lu MS, Liu KS, Liu EH, Chu JJ, Tsai FC, Lin PJ (2010) Traumatic pericardial effusion: impact of diagnostic and surgical approaches. Resuscitation 81:1682-1686

71. Hernández-Luyando L, González de las Heras E, Calvo J, López C, de la Puente H (1997) Posttraumatic tension pneumopericardium. Am J Emerg Med 15:686-687

72. Leibecke T, Stoeckelhuber BM, Gellissen J et al (2008) Posttraumatic and postoperative cardiac luxation: computed tomography findings in nine patients. J Trauma 64:721-726 